\title{
An observable prerequisite for the existence of persistent
}

\section{currents}

Jacob Szeftel ${ }^{1}$, Nicolas Sandeau ${ }^{2}$, and Michel Abou Ghantous ${ }^{3}$

1 ENS Cachan, LPQM, 61 avenue du Président Wilson, 94230 Cachan

2 Aix Marseille Univ, CNRS, Centrale Marseille, Institut Fresnel, F-13013 Marseille, France

American University of Technology, AUT Halat, Highway, Lebanon

the date of receipt and acceptance should be inserted later

\begin{abstract}
A classical model is presented for persistent currents in superconductors. Their existence is argued to be warranted because their decay would violate the second law of thermodynamics. This conclusion is achieved by analyzing comparatively Ohm's law and the Joule effect in normal metals and superconducting materials. Whereas Ohm's law applies in identical terms in both cases, the Joule effect is shown to cause the temperature of a superconducting sample to decrease. An experiment is proposed to check the validity of this work in superconductors of both types I and II.
\end{abstract}

PACS. 74.20.Mn Nonconventional mechanisms - 74.25.Bt Thermodynamic properties - 74.25.Fy Transport properties

\section{Introduction}

The prominent signature of superconductivity, i.e. the property to sustain persistent currents $[1,2,3]$ in vanishing electric field, has remained unexplained, since its discovery [4, as stressed by Ashcroft and Mermin[1] (see[1] p.750, $1^{\text {st }}$ paragraph, line 1) : the property for which the superconductors are named is the most difficult to extract from the microscopic (i.e. BCS[5]) theory. This is all the more dis- turbing, since the mainstream narrative on superconductivity relies heavily on the phenomenological equations, proposed by London[6] and Ginzburg and Landau[7], for which the existence of persistent currents is merely assumed.

In order to understand why this long-standing riddle has withstood every attempt $[8]$ at elucidating it so far, it is helpful to recall the basic tenets of electric conductivity in normal conductors [1. The applied electric field $E$ 
accelerates the electrons in the conduction band, which gives rise to a current $j$. Eventually, the driving force $\propto E$ is counterbalanced by a friction one $\propto j$, exerted by the lattice, as conveyed by Ohm's law :

$$
j=\sigma E \quad, \quad \sigma=\frac{c_{0} e^{2} \tau}{m},
$$

where $\sigma, c_{0}, e, m, \tau$ stand for the conductivity, the electron concentration, the electron charge, its effective mass, and the decay time of $j$ due to friction, respectively. Simultaneously, the work performed by the electric force is entirely transformed into heat, to be released in the lattice, through the Joule effect. As a consequence of Eq.(1), the observation [4] of $j \neq 0$ despite $E=0$ seemed indeed to suggest $\tau \rightarrow \infty \Rightarrow \sigma \rightarrow \infty$. However, it is wellknown nowadays that both $\tau, \sigma$ are finite, provided the measurement is carried out with an ac current, as emphasized by Schrieffer 9] (see 9 p.4, $2^{\text {nd }}$ paragraph, lines 9,10): at finite temperature, there is a finite ac resistivity for all frequencies $>0$. For instance, the conductivity, measured in $Y \mathrm{Ba}_{2} \mathrm{Cu}_{3} \mathrm{O}_{7}$ below the critical temperature $T_{c}$, has been reported[10,11] to be such that $\sigma \approx 10^{5} \sigma_{n}$, where $\sigma_{n}$ stands for the normal conductivity, measured just above $T_{c}$. Additional evidence is provided by commercial microwave cavity resonators, made up of superconducting materials, displaying a very high, albeit finite conductivity (see 3] lowest line in p.38). Finally, the observable consequences of finite $\sigma$, regarding the skin [12,13] and Meissner 6, 14] effects, have been discussed recently [15, 16] and the finite resistivity of superconductors has been ascribed solely to superconducting electrons 17] on the basis of susceptibility data.
Therefore the issue of persistent currents will be tackled here from quite different a starting point. Likewise we shall show how the very properties of the BCS state $[5$ cause the Joule dissipation to be thwarted in a superconductor, undergoing no electric field. This goal will be achieved by making a comparative study of Ohm's law and the Joule effect in normal and superconducting metals, based on Newton's law and the two laws of thermodynamics.

The outline is as follows : the conditions for a superconductor to be in thermal equilibrium are discussed in sections 2, while stressing the different properties of the BCS state [5] versus those of the Fermi gas [1]; Ohm's law and the Joule effect are studied in sections 3 and 4, respectively; a necessary condition for the existence of persistent currents is worked out in section 5, while an experiment, enabling one to check the validity of this analysis in superconducting materials of both kinds, is described in section 6. Our observable predictions will turn out to concur very well with a remark by De Gennes[18. The results of this work are summarized in the conclusion.

\section{The two-fluid model}

The conduction properties of a superconducting material will be analyzed within the two-fluid model $[2,3,9,18$. In this framework, the conduction electrons make up a homogeneous mixture, in thermal equilibrium, of normal and superconducting electrons, in concentration $c_{n}, c_{s}$, respectively. 
All of the electronic properties of the normal state are governed by the Fermi-Dirac statistics, and thence accounted for within the Fermi gas[1 model. In particular, its Helmholz free energy per unit volume $F_{n}$ depends on two parameters, the temperature $T$ and the Fermi energy $E_{F}$, defined[1,19] as the chemical potential of independent electrons, i.e. $E_{F}=\frac{\partial F_{n}}{\partial c_{n}}$.

By contrast, the BCS wave-function [5] describes the motion of superconducting electrons, as a many-body bound state, which entails that the BCS energy per unit volume $\mathcal{E}_{s}$ depends only on the concentration of superconducting electrons $c_{s}$. Because $\mathcal{E}_{s}$ is $T$-independent, the BCS state [5, unlike the Fermi gas, is inferred to carry no entropy [1,2,3], so that its free energy is equal to $\mathcal{E}_{s}$ (this property is confirmed experimentally by the weak thermal conductivity $1,2,3$, measured in superconductors, in marked contrast with the high one, typical of normal metals). Thus the chemical potential $\mu$ of the BCS state reads $\mu=\frac{\partial \mathcal{E}_{s}}{\partial c_{s}}$.

The equilibrium, achieved in the two-fluid model, stems from Gibbs and Duhem's law 19, which requires the free energy of the whole electron system $F_{e}=F_{n}\left(T, c_{n}\right)+$ $\mathcal{E}_{s}\left(c_{s}\right)$ to be minimum with respect to $c_{n}, c_{s}$, under the constraints $c_{n}+c_{s}=c_{0}\left(c_{0}\right.$ refers to the total concentration) and $T$ kept constant, and thence leads to

$$
E_{F}\left(T, c_{n}\right)=\mu\left(c_{s}\right)
$$

The peculiar properties of the Joule effect, taking place in a BCS state, will appear below to be solely determined by the $\operatorname{sign}$ of $\frac{\partial \mu}{\partial c_{s}}=\frac{\partial^{2} \mathcal{E}_{s}}{\partial c_{s}^{2}}$.

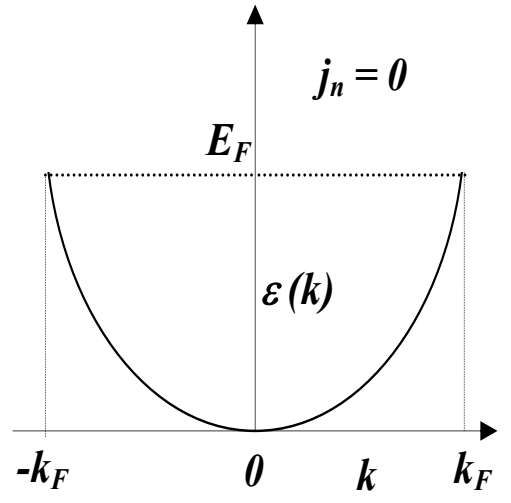

Fig. 1. schematic projected one electron dispersion $\epsilon(k)$ of occupied states $\left(\epsilon(k) \in\left[0, E_{F}\right]\right)$ for $j_{n}=0$ as a solid line

An early, phenomenological attempt 20], aimed at explaining the specific heat data, measured in superconducting materials, made use of Eq.(2) too. However our approach differs from that one, inasmuch as it refrains from assuming specific, but arbitrary expressions for $F_{n}\left(T, c_{n}\right), \mathcal{E}_{s}\left(c_{s}\right)$, so that our conclusions do not suffer from any loss of generality.

\section{Ohm's law}

Owing to Fermi-Dirac statistics and $T<<T_{F}=\frac{E_{F}}{k_{B}} \approx$ $3 \times 10^{4} K$ ( $k_{B}$ stands for the Boltzmann constant), the electrons in a normal metal make up a degenerate Fermi gas 1], for which each one-electron state, with energy ranging from the bottom of the conduction band up to $E_{F}$, is doubly occupied (due to the two spin directions), whereas those states with energy $>E_{F}$ remain empty. The corresponding one electron dispersion curve $\epsilon(k)$ has been projected onto the direction of the applied electric field $E$, as pictured in Fig,1. Since the electron velocity [1] is 


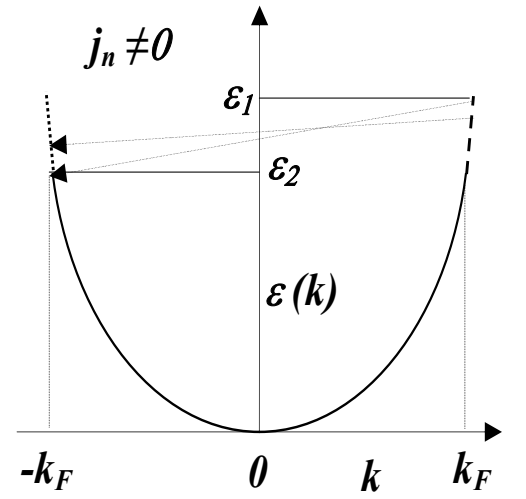

Fig. 2. schematic projected one electron dispersion $\epsilon(k)$ for $j_{n} \neq 0$ in the laboratory frame; the solid line represents most of electrons, which contribute nothing to $j_{n}$, whereas the dashed line corresponds to the few electrons responsible for $j_{n} \neq 0$; actually $j_{n}$ is proportional to the length of the dashed line but the tiny difference $\epsilon_{1}-\epsilon_{2}$ has been hugely magnified for the reader's convenience, that is $\epsilon_{1} \approx \epsilon_{2} \approx E_{F}$; the dotted line represents empty one electron states; the arrows illustrate electron transitions, from occupied (dashed line) back to empty (dotted line) states, driven by the friction force

equal to $\frac{\partial \epsilon(k)}{\hbar \partial k}$ and thanks to $\epsilon(k)=\epsilon(-k)$, the resulting current $j_{n}$ vanishes.

The applied field $E$ arouses a finite current $j_{n} \neq 0$ by accelerating $\delta c_{n}$ of electrons $\left(\delta c_{n}<<c_{n}\right)$ from their initial wave-vector $-k_{F}$ up to their final one $k_{F}$, with $k_{F}$ being such that $\epsilon\left(k_{F}\right)=E_{F}$, due to Pauli's principle. Therefore all electrons, contributing to $j_{n}$, have about the same velocity $v_{F}=\frac{\partial \epsilon\left(k_{F}\right)}{\hbar \partial k}$, so that the resulting current reads $j_{n}=2 \delta c_{n} e v_{F}$ (see the dashed line in Fig 2). Inversely, the friction force, exerted by the lattice on those electrons making up $j_{n}$, tends to bring $2 \frac{\delta c_{n}}{\tau_{n}}$ of electrons per unit time from $k_{F}$ back to $-k_{F}$, where $\tau_{n}$, showing up in Eq.(1) as $\tau$, represents the average time between two successive scattering events 1 (see the arrows pointing to the dotted line in Fig 2). As the momentum change rate, involved in this process, is equal to

$$
\frac{\delta p}{\tau_{n}}=-2 \frac{m v_{F} \delta c_{n}}{\tau_{n}}=-\frac{m}{e \tau_{n}} j_{n}
$$

Newton's law reads 15,16 , finally

$$
\frac{m}{e} \frac{d j_{n}}{d t}=c_{n} e E-\frac{m}{e \tau_{n}} j_{n}
$$

Because the inertial force $\frac{m}{e} \frac{d j_{n}}{d t}$ has been shown to be negligible [15, 16], the electric force $c_{n} e E$ and the friction one $-\frac{m}{e \tau_{n}} j_{n}$ cancel each other, so that Eq.(3) boils down to Ohm's law, as expressed in Eq.(1).

Ohm's law will be worked out now for a superconductor by proceeding similarly as hereabove. The $j_{s}=0$ superconducting state $\left(j_{s}\right.$ refers to the superconducting current) is assumed to consist in two subsets, each of them comprising the same number of electrons. It ensues, from the very properties of the BCS state[5], flux quantization and Josephson's effect [1,2,21, that the electrons in each subset are organized in pairs, moving in opposite directions with respective velocity $v_{s},-v_{s}$, which ensures $j_{s}=0$. The driving field $E$ causes $\delta c_{s}$ of electrons $\left(\delta c_{s}<<c_{s}\right)$ to be transferred from one subset to the other, which results into a finite current $j_{s}=2 \delta c_{s} e v_{s}$. The friction force is responsible for the reverse mechanism, whereby an electron pair is carried from the majority subset of concentration $c_{s}+\delta c_{s}$ back to the minority one of concentration $c_{s}-\delta c_{s}$. Hence if $\tau_{s}^{-1}$ is defined as the transfer probability per time unit of one electron pair, the electron transfer rate is equal to

$$
\frac{c_{s}+\delta c_{s}-\left(c_{s}-\delta c_{s}\right)}{\tau_{s}}=2 \frac{\delta c_{s}}{\tau_{s}} .
$$


Then Newton's law reads similarly as Eq.(3), valid for independent electrons

$$
\frac{m}{e} \frac{d j_{s}}{d t}=c_{s} e E-\frac{m}{e \tau_{s}} j_{s}
$$

As for independent electrons, the electric force $c_{s} e E$ and the friction force $-2 \frac{m v_{s} \delta c_{s}}{\tau_{s}}=-\frac{m j_{s}}{e \tau_{s}}$ cancel each other, which yields the searched result, identical to Eq.(1)

$$
c_{s} e E=\frac{m}{e \tau_{s}} j_{s} \Rightarrow j_{s}=\sigma_{s} E \quad, \quad \sigma_{s}=\frac{c_{s} e^{2} \tau_{s}}{m}
$$

Although Ohm's law displays the same expression for normal and superconducting metals as well, it should be noted that $\tau_{s}>>\tau_{n}[10,11$.

Finally note that the inter-electron forces, responsible for the binding energy of the BCS state with respect to the corresponding Fermi gas of same electron concentration and also for the two-electron scattering within the Fermi gas, do not show up in Eqs.(344). In order to understand this feature, let us consider two electrons labelled $i, j$. They exert the forces $\mathbf{f}_{i \rightarrow j}, \mathbf{f}_{j \rightarrow i}$ on each other, respectively. Due to $\mathbf{f}_{i \rightarrow j}+\mathbf{f}_{j \rightarrow i}=\mathbf{0}$, the net force, resulting from all $i, j$ pairs, vanishes and thence does not contribute to Ohm's law, although the inter-electron coupling will turn out to play a paramount role in the Joule effect.

\section{The Joule effect}

Because no electron contributes to $j_{n}$, but the few ones in concentration $2 \delta c_{n}$ with $\epsilon(k) \approx E_{F}$, showing up as the dashed line in Fig,2, they are also the only ones to be instrumental in the Joule effect. Besides all of them have the same velocity $v_{F}$. Thus, the well-known formula of the power released by the Joule effect, $\dot{W}_{J}=\frac{d W_{J}}{d t}$ ( $t$ refers to time), ensues from Ohm's law $j_{n}=\sigma_{n} E$, which implies that the friction force equals $2 \delta c_{n} e E$, as

$$
\dot{W}_{J}=2 \delta c_{n} e \mathbf{E} \cdot \mathbf{v}_{F}=\mathbf{E} \cdot \mathbf{j}_{n}=\frac{j_{n}^{2}}{\sigma_{n}}
$$

for which we have made use of $\mathbf{j}_{n}=2 \delta c_{n} e \mathbf{v}_{F}$.

The Joule effect takes place via two different processes in a superconductor. The calculation of the Joule power $\dot{W}_{1}$, released through process I, is identical to that one leading to Eq.(6)

$$
\dot{W}_{1}=\frac{m}{e \tau_{s}} \mathbf{j}_{s} \cdot \mathbf{v}=\frac{j_{s}^{2}}{\sigma_{s}}
$$

where $\mathbf{v}$ is the mass center velocity of superconducting electrons $\left(\Rightarrow \mathbf{v}=\frac{\mathbf{j}_{s}}{c_{s} e}\right)$ and advantage has been taken of Ohm's law in Eq.(5) to express the resulting friction force $\left(=\frac{m}{e \tau_{s}} \mathbf{j}_{s}\right)$, exerted on the mass center of superconducting electrons. The physical significance of Eq.(7) is such that the work $W_{1}>0$ performed by the driving force $\propto E$ is turned into heat by the friction force.

However, the calculation of the Joule power $\dot{W}_{2}$, released through process II, proceeds otherwise, because the work $W_{2}$, to be turned into heat by the friction force, is performed by the inter-electron forces, rather than the driving one, as seen for $W_{1}$ in Eq.(7). Accordingly, while any electron in a normal metal may lose, due to Pauli's principle, an energy randomly distributed from 0 up to $\epsilon_{1}-\epsilon_{2}$ (see Fig 21), conversely the corresponding internal energy change, experienced by the BCS electrons, due to the scattering of one electron pair, is uniquely defined, as will be shown hereafter. 
In case of $j_{s} \neq 0$, the chemical potential of majority (minority) electrons, characterized by the average velocity $v_{s}\left(-v_{s}\right)$ reads $\mu\left(c_{s}+\delta c_{s}\right)\left(\mu\left(c_{s}-\delta c_{s}\right)\right)$. During each elementary scattering process, a single pair is brought back from the majority subset to the minority one, which results into $\delta \mathcal{E}_{s}$, the energy lost by the BCS electrons to the lattice, reading

$$
\delta \mathcal{E}_{s}=\mu\left(c_{s}+\delta c_{s}\right)-\mu\left(c_{s}-\delta c_{s}\right)=2 \frac{\partial \mu}{\partial c_{s}} \delta c_{s}
$$

Since the transfer rate is equal to $2 \frac{\delta c_{s}}{\tau_{s}}$, the Joule power $\dot{W}_{2}=2 \frac{\delta c_{s}}{\tau_{s}} \delta \mathcal{E}_{s}$ reads finally, due to $\mathbf{j}_{s}=2 \delta c_{s} e \mathbf{v}_{s}$

$$
\dot{W}_{2}=4 \frac{\partial \mu}{\partial c_{s}} \frac{\delta c_{s}^{2}}{\tau_{s}}=\frac{j_{s}^{2}}{\sigma_{J}}, \quad \sigma_{J}=\frac{\left(e v_{s}\right)^{2} \tau_{s}}{\frac{\partial \mu}{\partial c_{s}}}
$$

The result in Eq.(8) is noteworthy in two respects :

- even though $\dot{W}_{2}$ is still proportional to $j_{s}^{2}$ as $\dot{W}_{1}$ in Eq.(7), the conductivity $\sigma_{s}$, deduced from Ohm's law in Eq.(15), differs from $\sigma_{J}$

$$
\sigma_{s}=\frac{c_{s} e^{2} \tau_{s}}{m} \neq \frac{\left(e v_{s}\right)^{2} \tau_{s}}{\frac{\partial \mu}{\partial c_{s}}}=\sigma_{J}
$$

- unlike $\sigma_{s}>0$, the sign of $\sigma_{J}$, which sets whether the Joule heat $W_{2}$ will flow from the conduction electrons towards the lattice ( $\Leftrightarrow W_{2}>0$ ), as is always the case in a normal conductor, or conversely will flow into the reverse direction $\left(\Leftrightarrow W_{2}<0\right)$, is to be determined by the sign of $\frac{\partial \mu}{\partial c_{s}}$. As a matter of fact, the searched criterion for the existence of persistent currents will be worked out by taking advantage of this peculiarity.

It is worth elaborating upon the significance of $\sigma_{s} \neq$ $\sigma_{J}$. The Joule power $\dot{W}_{J}$ reads in general

$$
\dot{W}_{J}=\sum_{i} \mathbf{f}_{i} \cdot \mathbf{v}_{i}
$$

where the sum is carried out on every electron in the conduction band, labeled by the index $i$, moving with velocity $\mathbf{v}_{i}$ and undergoing the friction force $\mathbf{f}_{i}$. Owing to Ohm's law, which implies that the resulting friction force equals $2 \delta c_{n} e \mathbf{E}$, and $\mathbf{v}_{i}=\mathbf{v}_{F}$ for all electrons contributing to $\mathbf{j}_{n}=2 \delta c_{n} e \mathbf{v}_{F}$, Eq. (9) can be recast, for a normal metal, as

$$
\dot{W}_{J}=\sum_{i} \mathbf{f}_{i} \cdot \mathbf{v}_{i}=\left(\sum_{i} \mathbf{f}_{i}\right) \cdot \mathbf{v}_{F}=2 \delta c_{n} e \mathbf{E} \cdot \frac{\mathbf{j}_{n}}{2 \delta c_{n} e}=\frac{j_{n}^{2}}{\sigma_{n}}
$$

which is seen to be identical to Eq.(6). Hence the fact, that the same conductivity $\sigma_{n}$ shows up in both expressions of Ohm's law $j_{n}=\sigma_{n} E$ and the Joule effect $\dot{W}_{J}=\frac{j_{n}^{2}}{\sigma_{n}}$, is realized to result from the typical property of a degenerate Fermi gas, that all electrons, contributing to $\mathbf{j}_{n}$, have the same and one velocity $\mathbf{v}_{F}$. Besides, Eq.(6) expresses also the fact that the Joule heat $W_{J}$ is equal to the work performed by the driving force $\propto E$. However this is no longer true for the BCS state, because of the additional contribution $W_{2}$, expressed in Eq. (8). Accordingly, in case of a BCS state, the whole Joule power, reads as

$$
\dot{W}_{J}=\dot{W}_{1}+\dot{W}_{2}=j_{s}^{2}\left(\sigma_{s}^{-1}+\sigma_{J}^{-1}\right) \Rightarrow W_{J} \neq W_{1}
$$

Finally it remains to be shown that $W_{2}=0$ in a normal metal. The time-derivative of the work done by the interelectron forces $\mathbf{f}_{i \rightarrow j}, \mathbf{f}_{j \rightarrow i}$ reads $\dot{W}_{i j}=\mathbf{f}_{i \rightarrow j} \cdot \mathbf{v}_{j}+\mathbf{f}_{j \rightarrow i} \cdot \mathbf{v}_{i}$. Meanwhile $\mathbf{f}_{i \rightarrow j}+\mathbf{f}_{j \rightarrow i}=\mathbf{0}$ and $\mathbf{v}_{i}=\mathbf{v}_{j}=\mathbf{v}_{F}$ imply that $\dot{W}_{i j}=$ 0. Q.E.D. 
5 Prerequisite for the existence of persistent

\section{currents}

The applied field $E$ gives rise to the total current $j=$ $j_{n}+j_{s}$, where $j_{n}=\sigma_{n} E$ and $j_{s}=\sigma_{s} E$, as required by Ohm's law. After $E$ has vanished, $j_{n}$ is quickly destroyed by the Joule effect. However whether $j_{s}$ will decay down to 0 or conversely will turn to a persistent current, will be shown hereafter to depend solely upon the sign of the whole Joule power $\dot{W}_{J}$, generated via processes I and II. In case of $E=0$, the kinetic energy, associated with $j_{s} \neq$ $0, \mathcal{E}_{K}=\frac{c_{s} m}{2} v^{2}=\frac{m}{2 c_{s} e^{2}} j_{s}^{2}$, due to $j_{s}=c_{s} e v$, is turned into heat by the friction force. The expression of $\dot{\mathcal{E}}_{K}$ is obtained, thanks to $j_{s}=2 \delta n e v_{s}$ and $\dot{v}=-\frac{2 \delta n v_{s}}{c_{s} \tau_{s}}$, as

$$
\dot{\mathcal{E}}_{K}=-c_{s} m v \dot{v}=-\frac{m}{c_{s} e^{2} \tau_{s}} j_{s}^{2}=-\frac{j_{s}^{2}}{\sigma_{s}}
$$

so that the expression of $\dot{W}_{1}=-\dot{\mathcal{E}}_{K}$ remains unaltered with respect to that one in Eq.(77) and finally we get the same expression as in the $E \neq 0$ case, i.e.

$$
\dot{W}_{J}=j_{s}^{2}\left(\sigma_{s}^{-1}+\sigma_{J}^{-1}\right) .
$$

If $\dot{W}_{J}>0$, the Joule effect will cause eventually $j_{s}=0$ and the associated kinetic energy will be converted into heat, to be dissipated in the lattice, as occurs in a normal metal. Inversely in case $\dot{W}_{J}<0$, which requires both $\sigma_{J}<0 \Leftrightarrow \frac{\partial \mu}{\partial c_{s}}<0$ (see Eq.(8) ) and $\sigma_{J}+\sigma_{s}>0$, the Joule heat is seen to be bound to flow from the lattice towards the superconducting electrons, which will cause the lattice temperature to decrease. However, since such a spontaneous cooling of the system, comprising all of electron and lattice degrees of freedom, which can furthermore exchange neither heat, nor work with the outer world due to $E=0$, would cause its whole entropy to decrease, and would thence be tantamount to violating the second law of thermodynamics, the searched criterion is deduced to say that persistent currents can be observed, only if both following conditions are fulfilled

$$
\frac{\partial^{2} \mathcal{E}_{s}}{\partial c_{s}^{2}}=\frac{\partial \mu}{\partial c_{s}}<0 \Rightarrow \sigma_{J}<0 \quad, \quad \sigma_{J}+\sigma_{s}>0
$$

That those conditions in Eqs.(10) are necessary, but by no means sufficient ones, can be understood by looking back at Eq.(2). The equilibrium of the mixture of normal and superconducting electrons will be stable provided

$$
\frac{\partial E_{F}}{\partial c_{n}}+\frac{\partial \mu}{\partial c_{s}}>0
$$

Both stable and instable cases are illustrated in Figs 34 , where $E_{F}\left(T, c_{n}\right), \mu\left(c_{s}\right)$ have been plotted versus $c_{n}, c_{s}$, respectively. Note that $\frac{\partial E_{F}}{\partial c_{n}} \approx \rho\left(E_{F}\right)^{-1}>0$ where $\rho(\epsilon)$ is the density of one electron states in the conduction band[1]. The infinite slope $\frac{\partial E_{F}}{\partial c_{n}}\left(c_{n} \rightarrow 0\right) \rightarrow \infty$ is then typical of a 3 dimensional van Hove singularity [1, associated with the bottom of the conduction band, where $\rho(\epsilon \rightarrow 0) \propto \sqrt{\epsilon}$. The inequality in Fig $3, E_{F}\left(T_{i}, c_{n}\right)<$ $E_{F}\left(T_{f}, c_{n}\right), \forall c_{n}$ with $T_{i}>T_{f}$, ensues from $\frac{\partial \rho}{\partial E_{F}}\left(E_{F}\right)>0$ via the Sommerfeld integral[1], which will be shown elsewhere to be another prerequisite for the occurrence of superconductivity. At last in case $c_{s} \rightarrow 0$, there is $\mathcal{E}_{s} \approx \frac{\epsilon_{c}}{2} c_{s}$ where $\epsilon_{c}$ refers to the Cooper pair energy [22, which entails that $\mu(0)=\frac{\partial \mathcal{E}_{s}}{\partial c_{s}}(0)=\frac{\epsilon_{c}}{2}$.

The experiment, to be discussed below, is aimed primarily at bringing evidence of the anomalous $\left(\sigma_{J}<0 \Rightarrow\right.$ $\left.\dot{W}_{J}<0\right)$ Joule effect, associated with a BCS state. Since 
every superconducting material is claimed here to be characterized by $\dot{W}_{J}<0$, the experimental procedure will look for evidence of the sample temperature being lowered by the Joule effect.

There are in general two ways to have a current flowing through any conductor, i.e. either directly by feeding an externally controlled, time-dependent current $I(t)$ into the sample, or indirectly by inducing the current $j_{s}(t)$ via a time-dependent magnetic field $H(t)$ according to Faraday's law [12. Though the latter has been overwhelmingly favored $[2,3,8,14,18$, so far in experiments involving superconductors, the former procedure should be given preference for two reasons :

- as the Meissner effect[16] gives rise to a spatially inhomogeneous current $j_{s}(t, r)$ with $r$ referring to the local coordinate inside the sample, the Joule power $\dot{W}_{J}(t, r)$ will thereby vary with $r$, whereas both $j_{s}(t), \dot{W}_{J}(t)$ will remain $r$-independent within the former procedure;

- because of an irreversible consequence 16 of the finite conductivity $\sigma_{s}$, there can be no one-to-one correspondence between the applied magnetic field $H(t)$ and $j_{s}(t, r)$, so that the current distribution remains unknown, by contrast with $j_{s}(t)=\frac{I(t)}{S}, \forall t(S$ refers to the area of the sample cross-section) within the former procedure.

\section{Experimental outlook}

Consider a thermally isolated, superconducting sample, Then $I\left(t>t_{M}\right)$ decreases from $I\left(t_{M}\right)=I_{c}\left(T\left(t_{M}\right)\right)$ back taken in its initial state $T_{i}=T(t=0)<T_{c}, I(t=0)=$ to $I\left(t_{f}\right)=0$, corresponding to the final state, reached at

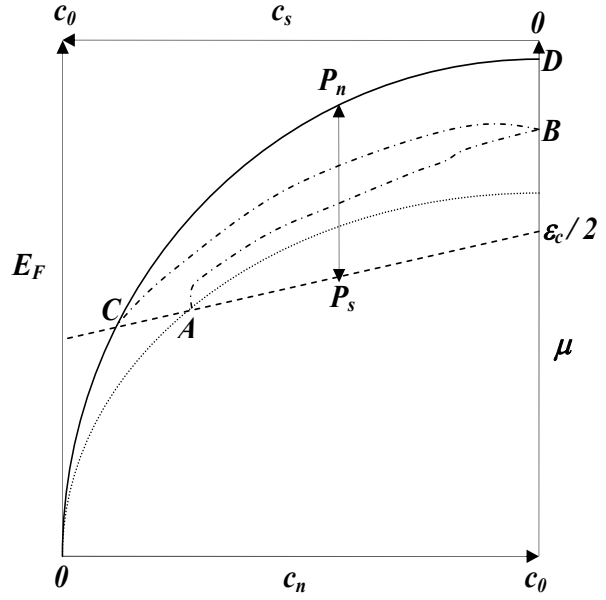

Fig. 3. schematic plots of $E_{F}\left(T_{i}, c_{n}\right), E_{F}\left(T_{f}, c_{n}\right)$ and $\mu\left(c_{s}\right)$ as dotted, solid and dashed lines, respectively, in case $\frac{\partial \mu}{\partial c_{s}}<0$, $\frac{\partial E_{F}}{\partial c_{n}}+\frac{\partial \mu}{\partial c_{s}}>0 ; \frac{\partial \mu}{\partial c_{s}}$ has been taken to be constant for simplicity; the origin $E_{F}=\mu=0$ is set at the bottom of the conduction band; the crossing points $A, C$ of $E_{F}\left(T_{i}, c_{n}\right), E_{F}\left(T_{f}, c_{n}\right)$, respectively, with $\mu\left(c_{s}\right)$, exemplify stable solutions of Eq.(2); the tiny differences $E_{F}\left(T_{f}, c_{n}\right)-E_{F}\left(T_{i}, c_{n}\right), E_{F}\left(T_{i}, c_{n}\right)-\mu\left(c_{0}-c_{n}\right)$ have been hugely magnified for the reader's convenience; the dashed-dotted line, linking $A, B, C$ together represents the adiabatic process, discussed in section VI; the points $P_{n}, P_{s}$ and the arrow linking them illustrate a superconducting-normal transition in progress (i.e. $c_{n}\left(T_{f}\right)<c_{n}\left(P_{n}\right)<c_{0}, 0<c_{s}\left(P_{s}\right)<$ $\left.c_{s}\left(T_{f}\right)\right)$, taking place at $T_{f}$, under the constraint $c_{n}+c_{s}=c_{0}$ $0, c_{n}(t=0)=c_{n}\left(T_{i}\right), c_{s}(t=0)=c_{s}\left(T_{i}\right)($ see $A$ in Fig 3$)$. Then let a direct current $I(t)$ flow through this sample. $I(t)$ grows from $I(0)=0$ up to its maximum value $I\left(t_{M}\right)$, reached at $t=t_{M}$, such that $I\left(t_{M}\right)=I_{c}\left(T\left(t_{M}\right)\right)$, with $I_{c}\left(T\left(t_{M}\right)\right)$ standing for the maximum persistent current [2] at $T=T\left(t_{M}\right)$, which causes the sample to go normal at $t=t_{M}$, i.e. $c_{n}\left(t_{M}\right)=c_{0} \Rightarrow c_{s}\left(t_{M}\right)=0($ see $B$ in Fig 3$)$. 


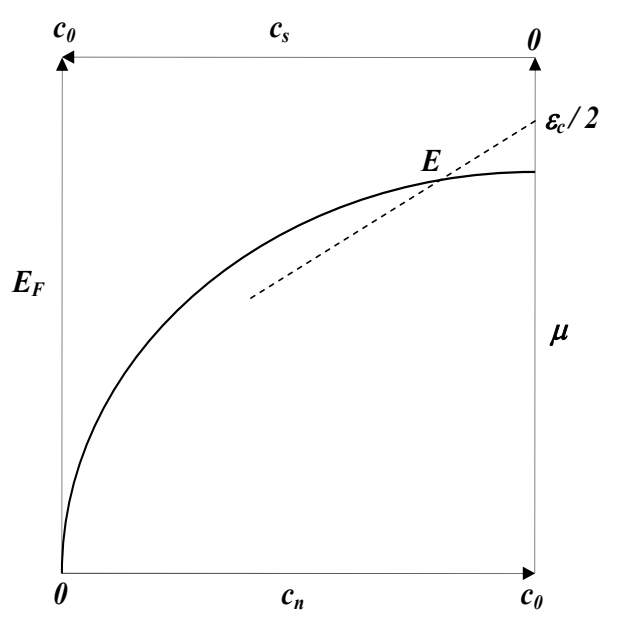

Fig. 4. schematic plots of $E_{F}\left(T, c_{n}\right)$ (solid line) and $\mu\left(c_{s}\right)$ (dotted line) in case $\frac{\partial \mu}{\partial c_{s}}<0$ and $\frac{\partial E_{F}}{\partial c_{n}}+\frac{\partial \mu}{\partial c_{s}}<0$; the crossing point $E$ of $E_{F}\left(T, c_{n}\right)$ with $\mu\left(c_{s}\right)$ represents an instable solution of Eq.(2)

$t=t_{f}$ and characterized by $T_{f}=T\left(t_{f}\right), I\left(t_{f}\right)=0, c_{n}\left(t_{f}\right)=$ $c_{n}\left(T_{f}\right), c_{s}\left(t_{f}\right)=c_{s}\left(T_{f}\right)($ see $C$ in Fig 3$)$.

The work $W\left(t_{f}\right)$, performed by the external electric field during the thermodynamical process, described hereabove and pictured as a dashed-dotted line in Fig 3 is then given by

$$
W\left(t_{f}\right)=\int_{0}^{t_{f}} U(t) I(t) d t
$$

with $U(t)$ designating the measured voltage drop across the sample. Since the sample is thermally isolated, applying the first law of thermodynamics to the system, comprising the independent and superconducting electrons and the lattice, driven from $A$ to $C$ via $B$ through an adiabatic process, yields then

$$
Q_{2}=\int_{T_{i}}^{T_{f}}\left(C_{\phi}(T)+C_{s}(T)\right) d T-W\left(t_{f}\right)
$$

with $W\left(t_{f}\right)$ being defined in Eq.(11). $C_{\phi}(T), C_{s}(T)$ stand for the respective contributions [1] to the specific heat of the phonons (Debye), which is $I$ independent, and of the conduction electrons, the latter being measured at $T \leq$ $T_{c}, I=0$. Then the integral over $T$ represents the difference in internal energy of the thermodynamical system, defined above, between $T_{i}$ and $T_{f}$. Besides, $Q_{2}=$ $V \int_{0}^{t_{f}} \frac{j_{s}^{2}(t)}{\sigma_{J}} d t=\frac{V}{S^{2}} \int_{0}^{t_{f}} \frac{I^{2}(t)}{\sigma_{J}} d t$ and $V$ are the Joule heat released via process II, and the sample volume, respectively $\left(j_{s}(t)\right.$, being $r$-independent, warrants $j_{s}(t)=\frac{I(t)}{S}, \forall t$ and $\left.Q_{2} \propto V\right)$.

As, due to $\sigma_{J}<0$ and $\sigma_{J}+\sigma_{s}>0$ (see Eqs.(10) $)$, the Joule effect is expected to cool down the sample, we predict that Eq.(12) will be fulfilled with $Q_{2}<0$ and $\frac{d T}{d t}\left(t \in\left[0, t_{f}\right]\right)<0 \Rightarrow T_{f}<T_{M}<T_{i}$, in full agreement with a remark by De Gennes 18] (see 18] footnote in p.18): if one passes from the superconducting state to the normal one in a thermally isolated specimen, the temperature of the sample decreases. Although this experiment could be done as well with $I\left(t_{M}\right)<I_{c}\left(T\left(t_{M}\right)\right)$, the condition $I\left(t_{M}\right)=I_{c}\left(T\left(t_{M}\right)\right)$ secures the largest $T_{i}-T_{f}$, because it maximizes $\left|j_{s}(t)\right|$ and thence $\left|\dot{W}_{2}\right|$.

Furthermore, the low value of $T_{c}$, encountered in first kind superconductors, ensures that $C_{s}\left(T \leq T_{f}\right)$ is known accurately. Conversely, for second kind superconductors, which includes all high- $T_{c}$ compounds, $C_{s}(T)$ is negligible 1] with respect to $C_{\phi}(T)$, so that Eq.(12) gets simpler

$$
Q_{2} \approx \int_{T_{i}}^{T_{f}} C_{\phi}(T) d T-W\left(t_{f}\right)
$$

Due to $C_{\phi}(T)$ being $I$ independent, unlike $C_{s}(T)$, taking the time derivative of Eq.(13) yields in addition

$$
\frac{V}{S^{2}} \frac{I^{2}(t)}{\sigma_{J}(t)}=C_{\phi}(T) \frac{d T}{d t}-U(t) I(t)
$$


which enables one to assess $\sigma_{J}(t)<0$ for $t \in\left[0, t_{f}\right]$ and thence to check $\sigma_{J}(t)+\sigma_{s}(t)>0$, the necessary conditions for the existence of persistent currents (see Eqs.(10)), provided $\sigma_{s}$ has been measured independently [10,11] (the $t$ dependences of $\sigma_{s}=\frac{c_{s} e^{2} \tau_{s}}{m}$ and $\sigma_{J}=\frac{\left(e v_{s}\right)^{2} \tau_{s}}{\frac{\partial \mu}{\partial c_{s}}}$ are both mediated by the $j_{s}$ dependence of $c_{s}(t)$, as demonstrated hereafter in the concluding section).

Although $T_{f}<T_{i}$ entails that the entropy of the twofluid system decreases, the second law of thermodynamics is thereby not violated, because the electrons remain coupled with the outer world via $I(t)$ during the experiment. At last, note that the state, illustrated by $B$ in Fig 3 refers to a metastable equilibrium, because the stable position at $T_{f}$ is rather inferred to be at $C$ in Fig 3 as required by Eq.(21). However, were the electron system to go spontaneously from $B$ to $C$, e.g. along the dasheddotted line, this process would result $\left[16\right.$ into $\frac{d j_{s}}{d t} \neq 0$, due to $j_{s} \neq 0$ at $B$ versus $j_{s}=0$ at $C$, while the accompanying Joule effect would give rise to a negative entropy variation $\Delta S_{B \rightarrow C}<0$, at odds with the second law of thermodynamics, as noted hereabove.

\section{Conclusion}

The anomalous Joule effect is characterized by $\sigma_{s} \neq \sigma_{J}$, i.e. the conductivity $\sigma_{s}$, deduced from Ohm's law, should differ from $\sigma_{J}$, the conductivity pertaining to the Joule power released through process II. It ensues solely from the inter-electron coupling, which causes the BCS electrons to gain the internal energy $\delta \mathcal{E}_{s}=W_{2}<0$ through process II at the expense of the lattice, while losing si- multaneously the kinetic energy $W_{1}>0$ through process I to the lattice, so that $W_{1}+W_{2}<0$ gives rise eventually to the cooling effect, embodied by Eqs.(12[13). Due to $W_{2}=0$ in a normal metal as shown above, the anomalous Joule effect can be observed solely for a many-body bound state, such as the BCS one. Likewise, the existence of persistent currents is warranted as a consequence of $\sigma_{J}<0$ and $\sigma_{J}+\sigma_{s}>0$ (see Eqs.(10)), because the resulting Joule dissipation $\dot{W}_{J}<0$ would run afoul at the second law of thermodynamics, which lends itself to an experimental check, as discussed above.

Besides, the property $\sigma_{s} \neq \sigma_{J}$ implies that Eq.(2) can never be fulfilled in presence of a persistent current $j_{s} \neq 0$. Here is a proof : consider the electron system in the equilibrium state, defined by $T=T_{f}, j_{s}=0$ and represented by $C$ in Fig 3, for which Eq.(2) is fulfilled. As $j_{s}$ grows from 0 up to its maximum value, the electron system shifts away from $C$ : the Fermi gas, represented by $P_{n}$ in Fig 3 moves, along the solid line, towards $D$, corresponding to the normal state $c_{n}=c_{0} \Rightarrow c_{s}=0$, while the BCS state, represented by $P_{s}$, goes, along the dashed line, towards the single Cooper pair state, characterized by $\mu\left(c_{s}=0\right)=\frac{\epsilon_{c}}{2}$, provided the sample remains connected to a heat bath at $T_{f}$. Meanwhile, whenever the thermodynamical state of the two-fluid system is represented by the pair $\left\{P_{n}, P_{s}\right\}$ in Fig 3. Eq.(2) is no longer fulfilled because of $E_{F}\left(T_{f}, c_{n}\left(P_{n}\right)\right)>\mu\left(c_{s}\left(P_{s}\right)=\right.$ $\left.c_{0}-c_{n}\left(P_{n}\right)\right)$, which demonstrates the first order nature of the $j_{s}$-driven superconducting-normal transition 2, 3, 9 , [18, by contrast with the second order transition, observed 
at $T_{c}$ with $j_{s}=0$, for which Eq.(2) is indeed fulfilled, i.e. $E_{F}\left(T_{c}, c_{0}\right)=\mu(0)=\frac{\epsilon_{c}}{2}$.

\section{References}

1. Ashcroft, N.W. and Mermin, N. D. Solid State Physics; ed. Saunders College, 1976.

2. Parks, R.D. Superconductivity; ed. CRC Press, 1969.

3. Tinkham, M. Introduction to Superconductivity; ed. Dover Books, 2004.

4. Onnes, H.K. The disappearance of the resistivity of mercury. Leiden Comm. 1911 122, 2.

5. Bardeen, J. and Cooper, L.N. and Schrieffer, J.R. Theory of superconductivity. Phys. Rev. 1957 108, 1175.

6. London, F. Superfluids vol.1; ed. Wiley, 1950.

7. Ginzburg, V.L. and Landau, L.D. On superconductivity theory Zh. Eksperim. i. Teor. Fiz. 1950 1064, 20.

8. Matricon J. and Waysand G., The Cold Wars, a History of Superconductivity; ed. Rutgers University Press, 2003.

9. Schrieffer, J.R. Theory of Superconductivity; ed. AddisonWesley, 1993.

10. Geshkenbein, V.B. and Vinokur, V.M. and Fehrenbacher, R. AC absorption in the high-T c superconductors: Reinterpretation of the irreversibility line. Phys. Rev. B 1991 43, 3748 .

11. Samarappuli, S. and Schilling, A. and Chernikov, M.A. and Ott, H.R. and Wolf, Th. Comparative study of AC susceptibility and resistivity of a superconducting YBa2Cu3O7 single crystal in a magnetic field. Physica C 1992 201, 159-165.

12. Jackson, J.D. Classical Electrodynamics; ed. John Wiley, 1998.
13. Born, M. and Wolf, E. Principles of Optics; ed. Cambridge University Press, 1999.

14. Meissner, W.and Ochsenfeld, R. Ein neuer effekt bei eintritt der supraleitfähigkeit. Naturwiss. 1933 21, 787-788.

15. Szeftel, J. and Sandeau, N. and Khater, A. Study of the skin effect in superconducting materials. Phys.Lett.A 2017 381, 1525-1528.

16. Szeftel, J. and Sandeau, N. and Khater, A. Comparative Study of the Meissner and Skin Effects in Superconductors. Prog.In.Electro.Res.M 2018 69, 69-76

17. Szeftel, J. and Abou Ghantous M. and Sandeau, N. Revisiting low-frequency susceptibility data in superconducting materials. Prog.In.Electro.Res.L 2019 81, 1-8

18. De Gennes, P.G. Superconductivity of Metals and Alloys; ed. Addison-Wesley, Reading, MA, 1989.

19. Landau, L.D. and Lifshitz, E.M. Statistical Physics; ed. Pergamon Press, London, 1959.

20. Gorter, C.J. and Casimir, H.B.G. The thermodynamics of the superconducting state. Z. tech. Phys 1934 15, 539542.

21. Josephson, B. D. Possible new effects in superconductive tunnelling. Phys. Letters 1962 1, 251-253.

22. Cooper, L.N. Bound electron pairs in a degenerate Fermi gas. Phys. Rev. 1956 104, 1189. 